rent stages of development. Int J Epidemiol. 2006; 35(1):112-3.)

\section{Revisión sistemática sobre el efecto de Plasmodium falciparum en la función cognoscitiva}

La infección por Plasmodium falciparum afecta a alrededor de 500 millones de personas en el mundo y causa 2,7 millones de muertes cada año. Esta es una de las principales especies causantes de malaria y al parecer puede causar daños al sistema nervioso central. Cada día gana más respaldo la hipótesis de que la infección grave por $P$. falciparum daña de manera más o menos persistente la capacidad cognoscitiva del enfermo, aunque no se sabe en qué medida esto pueda ocurrir cuando la enfermedad es menos grave. Este cuadro se hace aun más confuso por los diferentes criterios empleados para clasificar la gravedad de la enfermedad y por los diversos factores de riesgo que se han propuesto.

Los autores de este trabajo realizaron una revisión sistemática de los efectos de $P$. falciparum sobre la cognición, en particular la relación entre el parásito y la función cognoscitiva y las posibles diferencias en el deterioro neurológico y cognoscitivo en los niños y en los adultos.

La búsqueda abarcó todo lo publicado hasta agosto de 2005 y referido en MEDLINE (desde 1966), EMBASE (desde 1980) y PsycINFO (desde 1987). Los manuscritos se identificaron mediante la combinación de términos y palabras clave o por los títulos temáticos. Se revisaron las referencias bibliográficas de los artículos encontrados y las tesis de doctorado. Finalmente se analizó el texto completo de 25 documentos, de los cuales se descartaron 7 por no emplear pruebas estandarizadas para la población estudiada o controles adecuados o por no diferenciar claramente los resultados en niños y adultos.

En los niños, las formas graves de malaria estuvieron asociadas con un mayor grado de daño neurológico y cognoscitivo. En los adultos se encontraron deficiencias cognoscitivas relacionadas con la atención, la memoria y el lenguaje, pero no con la percepción visual o espacial o las funciones ejecutivas.

Aún no se conoce el mecanismo exacto mediante el cual el parásito $P$. falciparum causa el daño neurológico o cognoscitivo. La malaria puede influir negativamente en la cognición de manera indirecta, a través de una mala nutrición, la ausencia a la escuela o entorpeciendo el desarrollo psicosocial del enfermo. Sin embargo, hay pruebas contundentes de que la malaria cerebral causa trastornos como resultado de los daños directos que ocasiona en el cerebro. Trastornos específicos, como la disfunción del hipocampo y la lesión de la materia blanca subcorti- cal, pueden provocar alteraciones de la memoria y del lenguaje. Un estudio señaló la posibilidad de que la malaria cerebral provoque daños estructurales en la materia blanca del cerebro. Según otra hipótesis, las neurotoxinas liberadas por los glóbulos rojos infectados pueden dañar las áreas corticales del cerebro y así menoscabar las funciones cognoscitivas del enfermo. Como la comprensión del lenguaje, el aprendizaje y el razonamiento se ven mediados por la memoria, es posible que el daño neurológico y cognoscitivo provocado por la malaria esté relacionado con esta zona del cerebro.

A pesar de algunas limitaciones de este estudio, los resultados indican que la malaria puede provocar deficiencias en la atención, la memoria, las habilidades visuales y espaciales, el lenguaje y las funciones ejecutivas, tanto a corto como a largo plazo. Estos daños pueden observarse en casos de malaria cerebral por $P$. falciparum, pero también en formas menos graves de la enfermedad. La información disponible indica que es posible que este parásito afecte al cerebro en su conjunto y no de forma localizada. Al parecer, la edad en que ocurre la infección y el número de reinfecciones pueden ser variables que determinan la magnitud del daño neurológico y cognoscitivo.

El hecho de que cada año se infecten más de 500 millones de personas con este parásito hace pensar que la malaria puede ser una de las causas más frecuentes de trastorno cognoscitivo en el mundo. (Kihara M, et al. The effect of Plasmodium falciparum on cognition: a systematic review. Trop Med Int Health. 2006;11(4):386.)

\section{Factores sociodemográficos y ambientales asociados con el desmedro infantil en zonas rurales de Guatemala}

El retraso en el crecimiento o desmedro está asociado con el incremento de la morbilidad y la mortalidad en los niños y se calcula que alrededor de 182 millones de niños de países en desarrollo presentan este trastorno. A pesar del carácter multifactorial de las causas del desmedro, se ha documentado el papel causal que desempeñan la desnutrición y las infecciones frecuentes durante la infancia. Las deficientes condiciones de saneamiento y de higiene en el hogar, la mala situación socioeconómica, las diarreas frecuentes y el déficit de agua potable también pueden contribuir al desmedro. La incidencia de más de uno de estos factores aumenta el riesgo de padecerlo.

$\mathrm{Al}$ menos $50 \%$ de los niños de 30 a 35 meses de edad en Guatemala presentan una relación tallaedad por debajo de la línea de corte de desmedro. Se calcula que hasta $74 \%$ de los niños menores de 5 
años que viven en las regiones montañosas del país pueden presentar retraso en su crecimiento.

En este trabajo se identifican los factores socioeconómicos y ambientales asociados con el desmedro en los niños guatemaltecos. Se realizó un estudio transversal con 131 niños de 30 a 80 meses de edad que vivían en las comunidades rurales de Ciénaga Grande y El Pajón entre enero y marzo de 2001. Se excluyó a los niños que habían tenido diarrea (más de dos deposiciones líquidas en un día) en las $48 \mathrm{~h}$ previas a la toma de la muestra de heces o que tomaron algún antibiótico o antiparasitario en los 30 días previos.

Las muestras de heces y las mediciones antropométricas (talla y peso) se tomaron en instalaciones escolares. La persona encargada del cuidado directo del niño respondió a un breve cuestionario autoadministrado sobre las características sociodemográficas de la familia, los factores ambientales y del hogar que pudieran estar asociados con la transmisión de infecciones intestinales e influir en el patrón de crecimiento del menor, y la frecuencia y gravedad de los episodios de diarreas. La mayoría de los factores ambientales estuvieron relacionados con las características domésticas y socioeconómicas. La infección por Giardia lamblia se detectó mediante un sistema comercial con coproantígenos (AlexonTrend, Ramsey, Minnesota, Estados Unidos).

La prevalencia general de desmedro, bajo peso y deterioro progresivo en la población estudiada fue de $34,4 \%, 7,6 \%$ y $0,8 \%$, respectivamente. Los niños cuidados por personas analfabetas presentaron una probabilidad cinco veces mayor de tener desmedro (razón de posibilidades: 5,0; intervalo de confianza de $95 \%$ : 1,37 a 16,67) que los niños cuyos cuidadores sabían leer y escribir. Los niños que vivían con 4 niños o más en el mismo hogar tuvieron una probabilidad tres veces mayor de presentar desmedro (razón de posibilidades: 2,86; intervalo de confianza de 95\%: 1,17 a 7,14) que los niños que vivían en ambientes menos hacinados.

El análisis multifactorial de la varianza, con todas las variables estudiadas incorporadas al modelo, confirmó que solo las dos variables indicadas en el párrafo anterior permitían predecir el desmedro en los niños de la población estudiada $(P=0,02$ y $P=0,01$, respectivamente). A pesar de que beber agua de fuentes poco seguras y desechar inadecuadamente los residuos sanitarios se asociaron con el desmedro según el análisis unifactorial, esta relación no resultó estadísticamente significativa cuando se aplicó el análisis multifactorial.

Estos resultados demuestran la importancia del nivel educacional de los cuidadores directos de los menores y del número de residentes del hogar con el desmedro infantil. Estos factores deben tomarse en cuenta cuando se diseñen programas educativos de salud o intervenciones dirigidas a evitar el retraso del crecimiento en zonas rurales. (Sereebutra $\mathrm{P}$, et al. Sociodemographic and environmental predictors of childhood stunting in rural Guatemala. Nutr Res. 2006;26(2):65-70.)

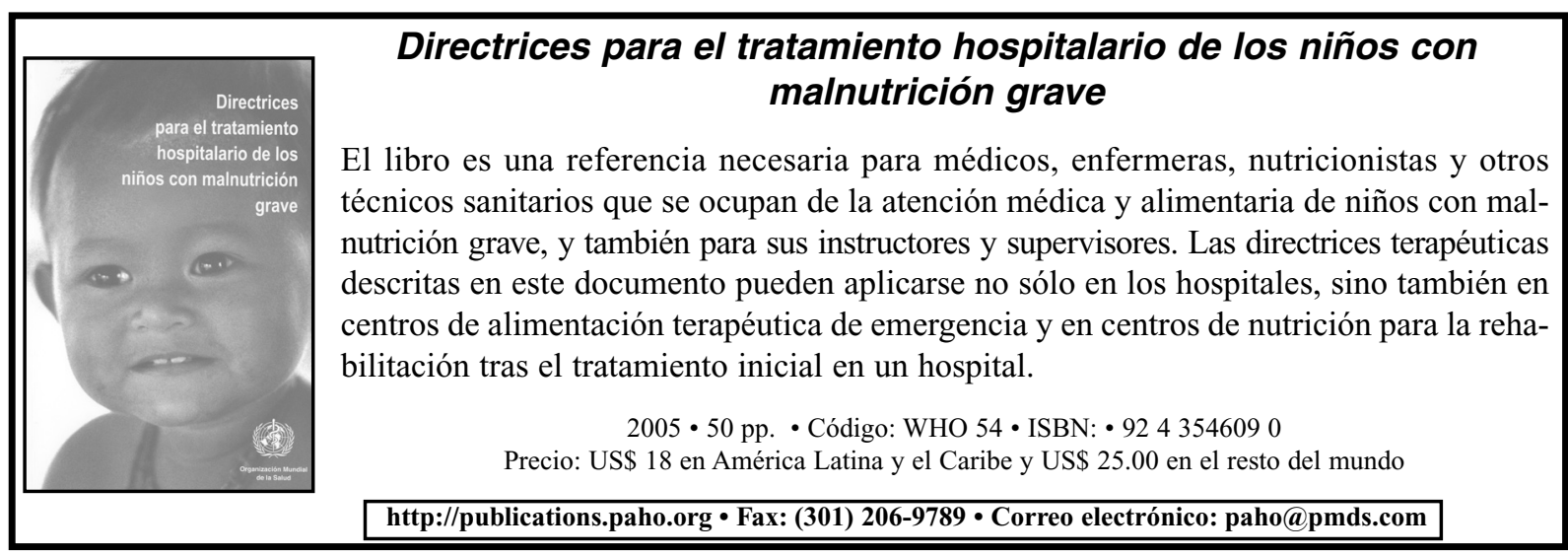

